\title{
Status of Cuore experiment and last results from Cuoricino
}

\author{
Elena Guardincerri* on behalf of the Cuore collaboration \\ Istituto Nazionale di Fisica Nucleare, Genova \\ E-mail: elena.guardincerri@ge.infn.it
}

\begin{abstract}
CUORE is a cryogenic-bolometer detector consisting of $988 \mathrm{TeO}_{2}$ crystals, $750 \mathrm{~g}$ each, operated at a temperature of $\sim 8 \mathrm{mK}$, currently under construction in the underground Laboratori Nazionali del Gran Sasso. Its goal is to search for neutrinoless double beta decays with a sensitivity to the effective neutrino mass as low as a few tens of meV. CUORICINO, its pilot experiment, has proven the feasibilty of CUORE setting moreover the current lower limit on the lifetime of ${ }^{130} \mathrm{Te}$ against neutrinoless double beta decay: we report on the up-to-date CUORICINO results and discuss the prospects for CUORE.
\end{abstract}

European Physical Society Europhysics Conference on High Energy Physics, EPS-HEP 2009, July 16 - 222009

Krakow, Poland

\footnotetext{
${ }^{*}$ Speaker.
} 


\section{Introduction}

The recent observations of neutrino oscillations by atmospheric[5], solar[2], and reactor[1] neutrino experiments estabilished that neutrinos are massive particles; the oscillation experiments have measured or constrained the elements of the neutrino mixing matrix and the values of the differences between the squared mass eigenvalues participating in the oscillations. The question whether neutrinos are Dirac or Majorana fermions is however still open and neutrinoless double beta decay $(0 v \beta \beta)$ is currently the only experimentally viable way to answer it. Besides this, the same process can probe the absolute neutrino mass scale by measuring the effective Majorana mass of the electron neutrino $m_{e e}$. The goal of the CUORE experiment is to measure $m_{e e}$ with a sensitivity in the $10-100 \mathrm{meV}$ range, where the spread is due to the uncertainty on the nuclear matrix element (see [3] and the references listed) involved in the determination of $m_{e e}$ from the ${ }^{130} \mathrm{Te}_{0 v \beta \beta}$ lifetime measurement. The feasibility of CUORE has been proved by its prototype, CUORICINO, which has been the most massive $0 v \beta \beta$ experiment currently running and whose performance will be discussed below.

\section{Principles of operation of CUORE and Cuoricino}

The search for $0 v \beta \beta$ is pursued by the bolometric technique: the detectors consist of $\mathrm{TeO}_{2}$ crystals operated at a temperature of $\sim 8 \mathrm{mK}$ inside a ${ }^{3} \mathrm{He} /{ }^{4} \mathrm{He}$ dilution refrigerator. The heat capacity of dielectric materials at this temperature is very low according to the Debye law, so that even tiny energy deposits in the crystals cause an appreciable rise in their temperature. Thermal pulses are recorded by neutron transmutated doped Ge thermistors glued on each crystals [6]. Among the few even-even nuclei candidates for $0 v \beta \beta$ decay, ${ }^{130} \mathrm{Te}$ has been chosen for its high transition energy (2527.518 $\pm 0.013 \mathrm{keV})$ and natural isotopic abundance $(33.87 \%)$. Besides achieving high energy resolutions, close to those obtained by Ge detectors, the bolometric technique provides the possibility to choose among different nuclei as sources for $0 v \beta \beta$ decay, thus allowing for a cross check in case of discovery.

\section{Cuoricino experiment}

\subsection{CUORICINO setup}

The CUORICINO detector[7] was an array of $62{ }^{130} \mathrm{TeO}_{2}$ bolometers operated in the Hall A of Laboratori Nazionali del Gran Sasso, Italy. The total sensitive mass was $40.7 \mathrm{~kg}$ and the mass of ${ }^{130} \mathrm{Te}$ was $\sim 11 \mathrm{~kg}$. The crystals were arranged in 13 planes: 11 of them consisted of four $5 \times 5 \times 5 \mathrm{~cm}^{3}$ crystals with a mass of $790 \mathrm{~g}$ each, 2 of them were made of nine $3 \times 3 \times 6 \mathrm{~cm}^{3}$ crystals whose mass was $330 \mathrm{~g}$. All crystals were made of natural tellurium except for four $3 \times 3 \times 6 \mathrm{~cm}^{3}$ $\mathrm{cm}^{3}$ ones: two of these are enriched in ${ }^{128} \mathrm{Te}$ with an isotopic abundance of $82.3 \%$ and two in ${ }^{130} \mathrm{Te}$ with an isotopic abundance of $75 \%$. Great care was taken to reduce radioactive contaminations and background sources at all stages of the detector construction and assembly: the crystals were grown from low radioactive materials at the Shangai Institute of Ceramics and shipped to Italy by sea to minimize cosmic activation. Once in Italy their surface was lapped with radiopure abrasives. 
To avoid external vibrations reaching the detectors, the tower was mechanically decoupled from the cryostat through a steel spring.

A $1.2 \mathrm{~cm}$ shield of Roman lead with ${ }^{210} \mathrm{~Pb}$ activity of $4 \mathrm{mBq} / \mathrm{kg}$ was framed around the array to reduce the backgrounds induced by contaminants on the thermal shields of the cryostat. The refrigerator itself was externally shielded by two layers of lead of $10 \mathrm{~cm}$ minimal thickness each. The background due to environmental neutrons was reduced by a layer of borated polyethylene of $10 \mathrm{~cm}$ minimum thickness. The refrigerator sits inside a Plexiglass anti-radon box flushed with clean $\mathrm{N}_{2}$ and a Faraday cage was framed around the whole setup to reduce electromagnetic interferences. The detector was calibrated every month by inserting two thoriated tungsten wires between the refrigerator and the external lead shield.

\subsection{Cuoricino results}

CUORICINO took data from February 2003 to June 2008. The background spectrum collected up to June
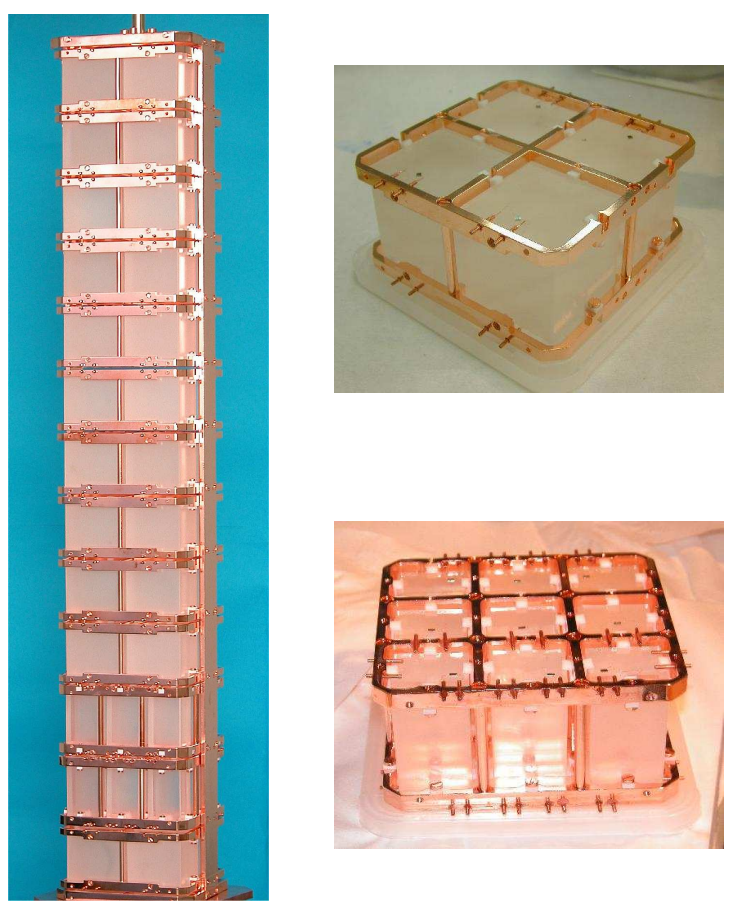

Figure 1: CUORICINO array (left) and details of the planes hosting the $5 \times 5 \times 5 \mathrm{~cm}^{3}$ crystals (top right) and $3 \times 3 \times 6 \mathrm{~cm}^{3}$ crystals (bottom right) 2008, corresponding to an exposure of 18 $\mathrm{kg}{ }^{130} \mathrm{Te} \cdot \mathrm{y}$, is shown in figure 2. The background level in the $0 v \beta \beta$ region is $0.18 \pm 0.01$ counts/(keV·kg.y) and the average energy resolution (FWHM), calculated on the ${ }^{208} \mathrm{Tl}$ gamma line at $2615 \mathrm{keV}$ is $7.8 \mathrm{keV}$ for the large crystals and $9.1 \mathrm{keV}$ for the small crystals. Apart from the ${ }^{60} \mathrm{Co}$ sum gamma line and the aforementioned ${ }^{208} \mathrm{Tl}$ line (not visible in figure 2), no peak is found near the $2527 \mathrm{keV}{ }^{130} \mathrm{Te} 0 v \beta \beta$ Q value. This allowed us to set a lower limit on the $0 v \beta \beta$ ${ }^{130} \mathrm{Te}$ hal-flife of $2.94 \times 10^{24}$ y at $90 \% \mathrm{CL}$. Depending on the nuclear matrix element calculation adopted, this can be translated into an upper limit on $m_{e e}$ in the range $m_{e e}<(0.21-0.70) \mathrm{eV}$. This constraint is currently the most restrictive for ${ }^{130} \mathrm{Te}$ and is comparable with the values obtained with Ge diodes.

\section{From CUORICINO to CUORE}

The CUORE detector[4] will consist of a cylindrical array of $988 \mathrm{TeO}_{2}$ bolometers arranged in 19 towers of 52 crystals each (figure 3), for a total mass of $\sim 741 \mathrm{~kg}, 203 \mathrm{~kg}$ of which will be ${ }^{130} \mathrm{Te}$. The detector will be operated at $T \simeq 8 \mathrm{~K}$ inside a dilution refrigerator in Hall A of Laboratori Nazionali del Gran Sasso, next to CUORICINO. In 5 years of running CUORE sensitivity to the 


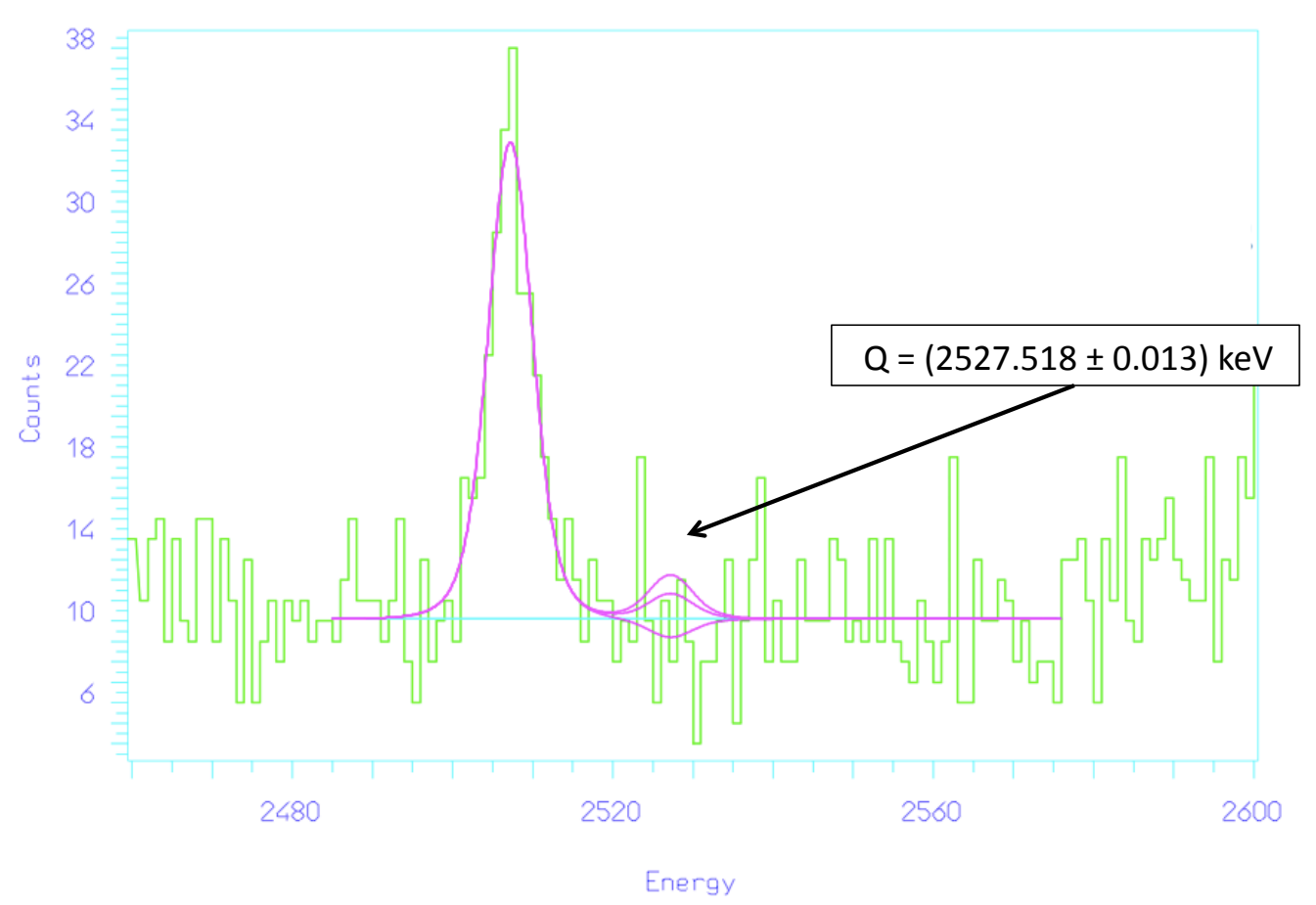

Figure 2: CUORICINO spectrum in the $0 v \beta \beta$ region

$0 v \beta \beta$ half-life of ${ }^{130} \mathrm{Te}$ will be $S_{0 v} \simeq 2.1 \times 10^{26}$ years: this will provide an upper limit on $m_{e e}$ in the range $0.024-0.083 \mathrm{eV}$. This sensitivity will be achieved by reducing the background in the $0 \mathrm{v} \beta \beta$ region to $B \simeq 0.01$ counts $/(\mathrm{keV} \cdot \mathrm{kg} \cdot \mathrm{y})$ and by improving the energy resolution to the level of $\Gamma(2.5$ $\mathrm{MeV})=5 \mathrm{keV}$. This requires careful materials selection, a slight improvement in the currently available material cleaning techniques, and an optimization in the mechanical decoupling of the bolometers from the surrounding environment in the detector design and assembly phase.

\section{CUORE prospects and current status}

Cuore construction is now proceeding in the Hall A of Laboratori Nazionali del Gran Sasso: the hut where the detector will be placed has been built and the construction of the clean room for the detector assembly will start shortly.

In parallel, the collaboration is also preparing Cuore 0, a prototype of Cuore consisting a single tower made of 52 already available Cuore crystals, prepared and assembled according to the Cuore standards and operated within Cuoricino cryostat. This detector will be the ultimate test of Cuore concept before Cuore start, and it is scheduled to take data in 2010-2011.

As mentioned in the previous section, the ultimate goal of CUORE is to measure or constrain $m_{e e}$ in the $\sim 0.024-0.083 \mathrm{eV}$ range. This will allow to probe part of the inverse and degenerate neutrino mass hierarchy pattern scenarios envisioned by neutrino oscillations experiments[8].

An improvement of this result could be obtained by either further reducing the background level or using ${ }^{130} \mathrm{Te}$ enriched crystals. Enriched $\mathrm{TeO}_{2}$ crystals have been already operated in CUORICINO making this latter option feasible. Assuming a 95\% enrichment and a background 
level of $B \simeq 0.01$ counts/(keV· $\mathrm{kg} \cdot \mathrm{y})$, the sensitivity of CUORE would be $S_{0 v} \sim 6.04 \times 10^{26}$ years in 5 years of running, yelding an upper limit on $m_{e e}$ in the range $11 \div 59 \mathrm{meV}$. The inverse hierarchy region could therefore be completely covered and the normal hierarchy parameter space partially spanned. CUORE is a joint European and American project. The detector construction is underway and data taking is scheduled to start in 2012 .

\section{Acknowledgements}

This work was performed under the auspices of the Italian Istituto Nazionale di Fisica Nucleare and the U. S. Department of Energy by the University of California, Lawrence Berkeley National Laboratory under Contract number KB0401022.

\section{References}

[1] S. Abe et al. Precision Measurement of Neutrino Oscillation Parameters with KamLAND. Phys. Rev. Lett., 100:221803, 2008.

[2] Q. R. Ahmad et al. Direct evidence for neutrino flavor transformation from neutral-current interactions in the sudbury neutrino observatory. Phys. Rev. Lett., 89:011301, 2002.

[3] F. T. Avignone.

The importance of accurate nuclear matrix elements in selecting, designing and analyzing double-beta decay experiments. AIP Conf. Proc., 942:1-7, 2007.

[4] J. Beeman et al. Cuore: An experiment to investigate for neutrinoless double beta decay by cooling $750-\mathrm{kg}$ of teo- 2 crystals at 10 -mk. AIP Conf. Proc., 850:1623-1626, 2006.

[5] S. Fukuda et al. Determination of solar neutrino oscillation parameters using 1496 days of super-kamiokande-i data. Phys. Lett., B539:179-187, 2002.

[6] E. E. Haller. J. Appl. Phys., 77:2857, 1995.

[7] S. Pirro et al. Further developments in the cuoricino experiment. Nucl. Instrum. Meth., A559:352-354, 2006.

[8] Alessandro Strumia and Francesco Vissani. Neutrino masses and mixings and... 2006.

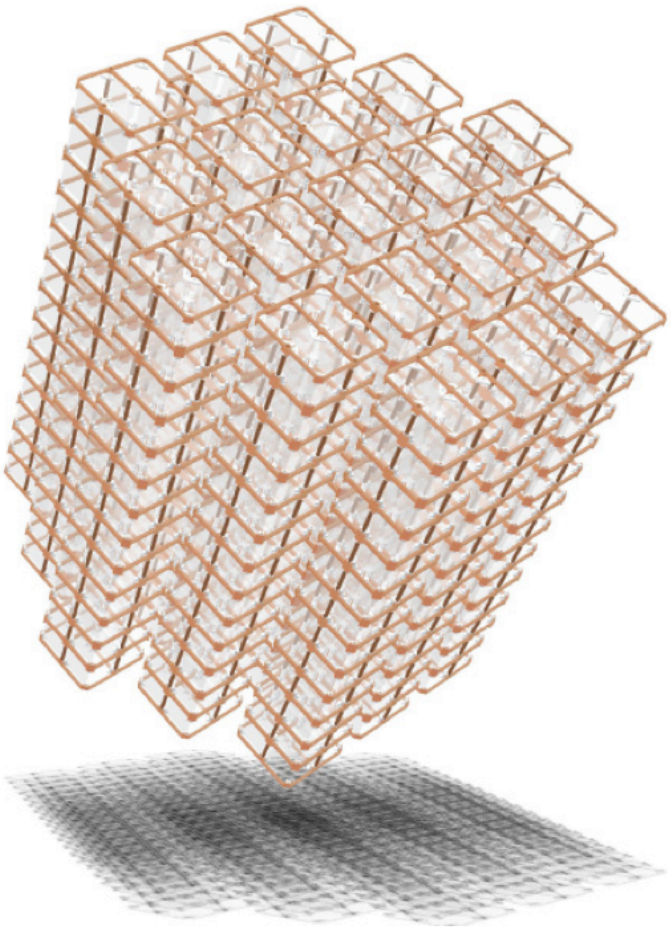

Figure 3: CUORE detector: the bolometers array is made of 19 CUORICINO like towers 